\title{
Acessibilidade dos homens a unidade básica de saúde
}

\author{
Accessibility of men to basic health unit \\ Accesibilidad de los hombres a la unidad básica de salud
}

\begin{abstract}
Sidney Mendes Pereira ${ }^{1 *}$, Francisca Eduarda Sousa Lima ${ }^{1}$, Francisco Igor dos Reis Gonçalves ${ }^{1}$, Nádia Bruna de Souza Silva Silva ${ }^{1}$, Wallison Queiroz dos Santos ${ }^{1}$, Patrícia Vidal Sousa ${ }^{1}$, Janete Brasil Torres ${ }^{1}$, Gilselene Costa de Oliveira ${ }^{1}$, Samuel Nascimento Morais ${ }^{1}$, Daiane Rodrigues Siqueira Rios ${ }^{1}$, Francisca Kelma Vieira dos Santos ${ }^{1}$, Rute Carvalho da Silva ${ }^{1}$, Elaine Irena Nogueira da Cruz ${ }^{1}$, Milena Martins da Costa e Silva ${ }^{1}$, Marcia Sousa Santos ${ }^{1}$
\end{abstract}

\section{RESUMO}

Objetivo: Analisar a acessibilidade do homem ao serviço de saúde. Método: Trata-se de uma pesquisa de campo com abordagem qualitativa, exploratória e descritiva, que foi realizada em uma unidade básica de saúde selecionada aleatoriamente na zona urbana da cidade de Caxias-MA no período de Agosto a Novembro de 2017. Foi utilizado para análise dos dados o Software Iramuteq, onde participaram do estudo 20 homens. Resultado: Os resultados foram processados a partir de um corpus no qual os segmentos de texto foram classificados em função da frequência dos vocábulos. Diante dessa classificação, surgiram 6 classes: classe 5: Motivo da procura do serviço de saúde. Classe 2: Percepção sobre serviços dispensados na unidade básica de saúde (UBS). Classe 3: Dificuldades enfrentadas ao acesso no serviço de saúde. Classe 4: Frequência do acesso do homem na unidade básica de saúde. Classe 6: Conhecimento dos homens sobre a UBS. Classe 1: A procura pelo atendimento nas unidades básicas de saúde. Conclusão: Pela análise das classe formuladas, conclui-se que a acessibilidade dos homens ás Unidades Básicas de Saúde é deficiente, pois os homens possuem conhecimento superficial sobre os serviços dispensados, e a ausência ou a frequência reduzida do mesmo, assim como o acesso a medicação em farmácias o próprio preconceito, a falta do auto cuidado proveniente de atribuições socioculturais, a insatisfação e a falta de confiança na assistência da atenção primária são fatores que dificultam a procura pelos homens á Unidades Básicas de Saúde.

Palavras-Chave: Saúde do homem, Acessibilidade, Unidade Básica de Saúde.

\begin{abstract}
Objective: To analyze the accessibility of the man to the health service. Method: This is a field research with a qualitative, exploratory and descriptive approach, which was performed at a randomly selected primary health unit in the urban area of the city of Caxias-MA from August to November 2017. It was used for data analysis the Iramuteq Software, where 20 men participated in the study. Results: The results were processed from a corpus in which the text segments were classified according to the frequency of the words. Facing this classification, there were 6 classes: class 5: Reason for the demand of the health service. Class 2: Perception of services dispensed at the basic health unit (UBS). Class 3: Difficulties faced with access to health services. Class 4: Frequency of access of the man to the basic health unit. Class 6: Knowledge of men about UBS. Class 1: The search for care in basic health units. Conclusion: Based on the analysis of the class formulated, it is concluded that the accessibility of men to Basic Health Units is deficient, since men have superficial knowledge about the services dispensed, and the absence or reduced frequency thereof, as well as access to medication in pharmacies the prejudice itself, the lack of self care from socio-cultural attributions, dissatisfaction and lack of confidence in the primary care assistance are factors that hinder the search for men to Basic Health Units.
\end{abstract}

Key words: Human health, Accessibility, Basic health Unit.

${ }^{1}$ Faculdade de Ciência e Tecnologia do Maranhão (FACEMA). *E-mail: thaylimacx@hotmail.com 


\section{RESUMEN}

Objetivo: Analizar la accesibilidad del hombre al servicio de salud. Método: Se trata de una investigación de campo con abordaje cualitativo, exploratorio y descriptivo, que se realizó en una unidad básica de salud seleccionada aleatoriamente en la zona urbana de la ciudad de Caxias-MA en el período de agosto a noviembre de 2017. Fue utilizado para análisis de los datos del Software Iramuteq, donde participaron del estudio 20 hombres. Resultado: Los resultados se procesaron a partir de un corpus en el que los segmentos de texto se clasificaron en función de la frecuencia de los vocablos. Ante esta clasificación, surgieron 6 clases: clase 5: Motivo de la demanda del servicio de salud. Clase 2: Percepción sobre servicios dispensados en la unidad básica de salud (UBS). Clase 3: Dificultades enfrentadas al acceso en el servicio de salud. Clase 4: Frecuencia del acceso del hombre en la unidad básica de salud. Clase 6 : Conocimiento de los hombres sobre la UBS. Clase 1: La demanda por la atención en las unidades básicas de salud. Conclusión: Por el análisis de las clases formuladas, se concluye que la accesibilidad de los hombres a las Unidades Básicas de Salud es deficiente, pues los hombres poseen conocimiento superficial sobre los servicios dispensados, y la ausencia o la frecuencia reducida del mismo, así como el acceso a la falta de autoestima proveniente de atribuciones socioculturales, la insatisfacción y la falta de confianza en la atención de la atención primaria son factores que dificultan la demanda por los hombres a las Unidades Básicas de Salud.

Palabras clave: Salud del hombre, Accesibilidad, Unidad Básica de Salud.

\section{INTRODUÇÃO}

Para Vieira et al. (2013), as unidades básicas de saúde consideram o ser humano em todas as perspectivas, seja na sua singularidade, complexidade, integralidade e inclusão sociocultural, evitando qualquer problema que possa agravar o bem-estar do ser humano. Para os autores, a equidade no ingresso da população masculina aos serviços de saúde é substancial.

As inúmeras mortes masculinas estão atreladas as especificidades biológicas, desigualdades sociais, valores culturais e as expectativas sociais para ambos os sexos, além dos conhecimentos dos profissionais acerca das necessidades dos homens para uma abordagem específica (MOURA et al., 2016).

Os fatores relacionados ao gênero limitam o acesso dos homens aos serviços de saúde, especificamente a atenção básica. Além disso, os homens procuram menos os serviços de saúde de atenção básica do que as mulheres e estes tendem a optar por serviços hospitalares, consultórios particulares e pronto atendimento ou serviços de emergência (OLIVEIRA et al., 2015).

Esta não procura do homem por atendimento na atenção básica, pode ser reflexo do papel social construído para o homem em que a masculinidade está atrelada a valores ou atributos como fortaleza, agressividade e competitividade que foram socialmente construídos, contribuindo para determinar, por exemplo, o perfil de morbimortalidade destes sujeitos (OLIVEIRA et al., 2015; CAVALCANTE et al., 2014).

Sendo assim, o presente estudo tem como objetivo analisar a acessibilidade do homem a unidade básica de saúde e investigar as dificuldades enfrentadas pelos homens ao acesso a unidade básica de saúde.

\section{METODOLOGIA}

O presente trabalho trata-se de um estudo de campo com abordagem qualitativa, exploratória e descritiva, foi realizado em unidades básicas de saúde na cidade de Caxias-MA, afim de compreender a acessibilidade dos homens a unidade básica de saúde.

O estudo de campo tem como foco uma comunidade, podendo ser geográfica ou não, já que se encaixa as comunidades de trabalho, lazer, de estudo, ou qualquer atividade humana. A pesquisa desenvolve-se com a observação do grupo estudado e por meios de entrevistas, análise de documentos, filmagem e fotografias para obter informações da interpretação do que ocorre no grupo estudado (GIL, 2007). 
Esta pesquisa foi realizada em uma unidade básica de saúde selecionada aleatoriamente, na cidade de Caxias, município do estado do maranhão. Caxias possui 36 unidades básicas de saúde na zona urbana e 11 na zona rural, a qual a totalidade de equipes de estratégia de saúde da família são 53 , essa quantidade é superior as unidades básicas de saúde por ter mais de uma equipe de ESF em algumas unidades.

A amostra foi composta por 20 homens, os participantes da amostra foram homens com idade entre $20 \mathrm{e}$ 50 anos, casados ou solteiros. O método de seleção da amostra foi de forma aleatória estando os indivíduos dentro das características mencionadas.

Após isso, foi realizada uma entrevista estruturada com 5 questões abertas, pois esta modalidade não induz o entrevistado a uma alternativa pretensiosa do entrevistador, além de oferecer maior abrangência de resposta do entrevistado. Foi utilizada um aparelho para gravação de áudio de toda a entrevista, a qual teve duração de dez a vinte minutos.

Todas as informações do estudo foram obtidas utilizando o seguinte instrumento de investigação: entrevista estruturada.

Compreende aquelas questões que são antecipadamente formuladas direcionadas para o que se quer pesquisar. Esse método possui questões diretivas, obtém do entrevistado respostas mais facilmente categorizadas, demanda certa liberdade para que o entrevistador possa captá-las adequadamente, sendo muito útil para levantamentos sociais (SEVERINO, 2014).

O projeto de pesquisa foi devidamente submetido a plataforma Brasil para ser avaliado, e posteriormente ao Comitê de Ética e Pesquisa, e o referido projeto foi aprovado com o $N^{\circ}$ de CAAE 71204917.3.0000.8007 e do PARECER 2.265.095 (ANEXOS). Esta pesquisa atende aos aspectos éticos que envolvem os estudos com seres humanos respaldada na resolução 466/2012 do conselho nacional de saúde, que institui as normas de pesquisas em saúde, através de uma declaração de compromisso assinada pelos pesquisadores e a carta de encaminhamento de projeto de pesquisa, encaminhada ao comitê de ética em pesquisa (CEP) da Faculdade de Ciências e Tecnologia do Maranhão - FACEMA. Os homens a serem entrevistados somente participarão da pesquisa após assinatura de um termo de consentimento livre esclarecido (TCLE APÊNDICE).

\section{RESULTADOS E DISCUSSÃO}

\section{Caracterização Dos Participantes}

Dos 20 participantes desta pesquisa, todos eram do sexo masculino com faixa etária entre 20 e 50 anos, cerca $40 \%$ doa participantes têm idade entre 20 a 30, 45\% apresentou idade entre 31 a 40 e 15\% têm entre 41 a 50, conforme o Tabela 1.

Tabela 1. Caracterização dos participantes em relação a faixa etária e nível de escolaridade

\begin{tabular}{cccc}
\hline FAIXA ETÁRIA & $20-30$ & $31-40$ & $41-50$ \\
PORCENTAGEM & $40 \%$ & $45 \%$ & $15 \%$ \\
ESCOLARIDADE & Fundamental & Médio & Superior \\
PORCENTAGEM & $20 \%$ & $50 \%$ & $30 \%$ \\
\hline Fonte: Elaboração própria & & &
\end{tabular}

Fonte: Elaboração própria 


\section{Motivo da procura do serviço de saúde}

O estudo demonstra que os principais motivos que levam o homem a unidade básica de saúde é o acompanhamento de parentes, como vacinação do filho, além de homens em faixa etária mais avançada, e em último momento, pegar algum encaminhamento ou fazer exames de rotina, no entanto, a minoria procura as unidades básicas de saúde (UBS) para prevenção de agravo a saúde, conforme as falas abaixo.

E 1: O principal... de ir ao posto...é o atendimento básico de prevenção, eu procuro ir pra prevenir, cuidar da minha saúde pra não se agravar, e... pra que não chegue, ou obrigado ir para as emergências dos hospitais, então... eu procuro o posto pra isso.

E 2: O motivo da minha ida ao posto é... pra fazer um checape, pra ver se tem algum problema.

E 3: Os motivos...quando vou levar algum parente principalmente filho pra tomar vacina, ou pra pegar algum encaminhamento pra outra especialidade.

E 4: Hoje em dia não tenho muitos motivos, mas algum tempo atrás o maior motivo de ir lá foi pra manter as vacinas do meu filho em dia.

E 5: Como eu não vou lá, eu não tenho nenhum motivo, só raramente acompanhando algum parente.

E 6:Os principais motivos é, a questão de vacinação mesmo, e os serviços odontológicos, só isso mesmo.

A forma de assistência da atenção primariamente vai muito além da dos aspectos preventivas, abrange também os aspectos curativos. Segundo o autor, dentre aqueles que mais frequentam a atenção básica se destaca as mulheres em comparação com homem, eles buscam esses serviços acompanhando algum ente querido e quando o problema já deve ser encaminhado para níveis mais avançados de resolução do problema (BARBOSA, 2014).

Embora o processo social e cultural tenha moldado o homem a ter comportamento distinto da mulher principalmente quanto a saúde, as transições e modificações dos papeis sociais vem contribuindo para o desempenho de tarefas que antes era somente feminina, e agora é realizada pelo homem. De acordo com os autores, os cuidados dos filhos, ou entes queridos, como locomoção desses até os ambientes de saúde, além do acompanhamento nesses locais feito por eles, tem aproximado os homens a terem uma visão diferenciada ao conceito de cuidado a saúde (SILVA; BODÓ; SILVA, 2013).

A procura da atenção primária de saúde se dá por homens com idade mais elevada, e isso está relacionado com os acometimentos de doenças crônicas. Para os autores, a praticidade da automedicação chama a atenção da população masculina por ser de fácil acesso e de baixo custo, não demandando tempo excessivo como os serviços de saúde (VIEIRA et al., 2013).

\section{Percepção sobre serviços dispensados na UBS}

Sobre a percepção dos homens sobre os serviços dispensados nas unidades básicas, os homens demonstram pouco conhecimento em relação a esses serviços, principalmente sobre as atividades preventivas para a promoção de saúde, de acordo com as falas abaixo.

E 1: Então! São as consultas as vacinas, e os atendimentos médicos para os idosos, além de curativos... só isso.

E 2: Eu entendo que esses serviços são serviços essenciais para a saúde daquela comunidade, no que elas necessitam, como um atendimento médico...e encaminhamentos para outros locais.

E 3: É... vacinação, marcação de exames, serviços odontológicos, são esses serviços ofertados no posto de saúde. 
E 4: Eu percebo que oferece vários serviços, exemplo lá se marca consulta, se faz uma extração de dente, um curativo e por mais é isso.

E 5: Oferece consultas médicas, é... curativos, pré-natal, além de dentistas e... outros a mais né.

Devido a dissociação dos homens das unidades básicas de saúde, poucos deles conhecem a real intenção da atenção básica, principalmente os serviços dispensados. O que faz ter opiniões especulativas e superficiais em relação a esse nível de atenção (SEPARAVICH; CANESQUI, 2013).

Um dos maiores problemas também enfrentadas na atenção básica, é causada por falta de informação quanto os serviços dispensados pela atenção básica. O usuário geralmente não reconhece as unidades básica como sendo a primeira a ser procurada de acordo com a necessidade. Isso pode gerar acumulação em outros níveis de atendimento enquanto a atenção primária fica sem demanda. A identificação desses serviços gera maior resolutividade e assim criando vinculo enquanto a qual serviço recorrer (OLIVEIRA; PEREIRA, 2013).

\section{Dificuldades enfrentadas ao acesso no serviço de saúde}

Em relação as dificuldades dos homens ao acesso das unidades básicas de saúde, os fatores relatados pelos pesquisados foram os aspectos culturais que envolve a virilidade masculina, o hábito de não ir com frequência a unidade básica de saúde afim de prevenir algum agravo a saúde.

Além disso, a facilidade de aquisição de medicamentos nas farmácias, a falta de confiança dos serviços prestados pelos profissionais das unidades, a burocracia pra marcar consultas, além da demora no atendimento, falta de material, além do horário inadequado pra marcar consulta. O tempo foi um dos fatores menos citados, em contrapartida a falta de confiança dos serviços e atendimento dispensados foi o que mais se destacou, de acordo com as falas a seguir.

E 1: Olha...seria a facilidade de acesso a medicamentos nas farmácias, e... o tempo, a questão do preconceito, o machismo de achar que tá tudo bem e... a dificuldade que também existe dentro dos postos de saúde, e achar que tudo tá bem, tudo vai passar.

E 2: O principal fator é de não ter o hábito de cuidar da saúde e também marcação de consultas demora demais.

E 3: Pra mim é exatamente a procura do serviço e não ter, como eu falei, as vezes você vai, mas não consegue, ou mesmo a falta de material pra algum procedimento.

E 4: A grande dificuldade pra é acordar três da manhã pra conseguir uma consulta, ou exame e também a questão do tempo de retorno ou o resultado do exame.

E 5: Eu geralmente não vou lá, poucas vezes já precisei, mas não confio muito nos serviços de lá, como hoje as farmácias disponibilizam os remédios eu sempre recorro a ela, além dos remédios caseiros que são bem acessíveis.

Os motivadores do homem ser menos frequentes nas unidades básicas de saúde geralmente são baseados nos aspectos culturais ou conforme as atitudes dele em relação a saúde. Porém não é exatamente a cultura, mas sim, a forma como ela se encontra impregnada na sociedade (MOREIRA; FONTES; BARBOSA, 2014).

A não procura por assistência à saúde pelos homens, o levam a formas diferenciadas para se tratar ou se beneficiar de efeitos medicamentosos. Para os autores, a automedicação é uma saída bastante utilizadas pelos homens para suprir suas necessidades, principalmente medicações que proporcionam ereção, isso coloca em anonimato circunstancias que poderiam constrange-los (FAROI et al., 2013).

A política nacional de atenção integral a saúde do homem enfrenta algumas dificuldades. Principalmente relacionada ao comportamento do homem, a busca por atendimento a problema de saúde em estágio avançado, e a percepção do fator curativo como sendo o principal para manter a saúde, ignorando a prevenção e promoção de agravos (RAMALHO et al., 2014). 
A referente política sobre a saúde do homem, mesmo sendo lançada em 2009, ela ainda não estar sendo executada na sua totalidade. Um dos motivos de maior mortalidade dos homens também está atrelado no pouco investimento e operacionalização das políticas públicas e na capacitação de profissionais e formação para atender as necessidades do homem (BIDINOTTO; SIMONETTI; BOCCHI, 2016).

A formação dos profissionais de saúde não pode ater-se unicamente para os aspectos curativos, ou de assistência de alta complexidade, a assistência deve ser primordialmente focalizada nos aspectos preventivos e promocionais, destacando estratégias para a educação em saúde pois desta forma contribuiria para a manutenção e proteção da saúde masculina. Os autores destacam que, a insatisfação do atendimento nos tais serviços, são fatores que contribuem para o afastamento dos homens, consequentemente, o mesmo não será indicado aos demais (ARRUDA; MATHIAS; MARCON, 2017).

\section{Frequência do acesso do homem na UBS}

Sobre a frequência, a maioria dos pesquisados relatam que vão raramente ou no máximo uma vez ao ano nas unidades básicas de saúde. Poucos relatam ir mais de uma vez, porém, sua ida está relacionada a acompanhamento de parentes e não para busca de atendimento próprio, conforme as falas a seguir.

E 1: Eu particularmente não vou as unidades básicas de saúde com frequência, mesmo passando por necessidade, e precisando ir por que o homem precisa, eu não tenho ido.

E 2: A minha frequência ao posto de saúde...uma vez ao ano.

E 3:Eu vou no posto de saúde somente uma vez por ano, mas tem ano que eu nem vou, vou poucas vezes por que tenho outras maneiras de...resolver os pequenos problemas.

E 4: Quase nunca, por que eu sempre tive um cuidado com minha saúde um pouco desleixado, só procuro mesmo em campanhas de vacinas mesmo.

E 5: Normalmente eu procuro a cada seis meses pra fazer um checape pra saber exatamente como se encontra minha saúde.

A concepção clássica do que o homem representa a serviço da família e da sociedade são fatores associados ao pesar do mesmo, o que reflete na insensibilidade do autocuidado causando dissociação e menor frequência às unidades básica de saúde. A masculinidade naturalmente fornece um conjunto de características protetora e que seu maior alicerce é sua robustez (SILVA; BODÓ; SILVA, 2013).

Os homens geralmente buscam atendimento em situações avançadas de agravo a saúde, ou quando a patologia começa a manifestar sinais e sintomas. De acordo com os autores, a insatisfação do atendimento é um dos problemas enfrentados pelos homens quando buscam a atenção primária de saúde, principalmente a morosidade a qual essa assistência demanda (ARRUDA; MATHIAS; MARCON, 2017).

Em comparação com as mulheres os homens não usam com frequência as unidades básicas de saúde. Para os autores, eles estão mais preocupados com a cura do problema e não com a prevenção, o que faz aumentar o quantitativo de pacientes masculinos em serviços de saúde de alta complexidade ou centros especializados (MOURA et al., 2014).

\section{Conhecimento dos homens sobre a UBS}

Em relação ao conhecimento dos homens sobre as unidades básicas de saúde, o estudo demonstra que os homens não sabem o real papel da unidade básica de saúde, e confundem o processo curativo com o preventivo que é o principal papel da UBS. No entanto, a minoria consegue definir com dificuldade a unidade básica de saúde, conforme as falas abaixo.

E 1: Não sei se seria o correto, mas...na minha visão, vejo um posto de saúde como uma linha de encaminhamento para as pessoas que...por um problema de... enfermidade procura seus recursos. E acredito que foram implantados os postos de saúde nos bairros na intenção de facilitar o acesso de quem está passando por problemas. 
E 2: É uma unidade que visa aproximar o hospital daquelas pessoas que tem mais dificuldade de locomoção, e também facilitar o atendimento em algumas áreas da saúde.

E 3: É um local destinado a tratar as pessoas e distribuir melhor os pacientes para não lotar os hospitais.

E 4: Rapaz o posto de saúde é...eu acho um local assim que...que é pra ajudar várias pessoas que está doente e... e dão vários tipos de remédios, pra ajudar assim... a saúde.

E 5: É um estabelecimento onde as pessoas vão se tratar e manter pra melhorar a saúde.

Os papeis masculinos desenvolvido durante sua vivência enquanto um ser inserido dentro de um grupo social, traz consigo responsabilidade como pai como marido e provedor familiar, sem falar no fator sexual onde não há igualdades. As perspectivas masculinas diante de condições pré-concebidas delimitam o conhecimento sobre a atenção primária e dificulta uma compreensão por parte do homem sobre autocuidado (GOMES et al., 2016).

Os fatores relacionados ao gênero limitam o acesso dos homens aos serviços de saúde, especificamente a atenção básica, isso porque não compreendem seu funcionamento, além de estereótipos culturalmente construído no que diz respeito a sua preponderância em relação as mulheres. Ainda para os autores, as unidades básicas de saúde são reconhecidas pelos homens como ambientes femininos, as questões decorrentes dessas posturas culminam em preocupações na saúde pública (VIEIRA et al., 2013).

\section{A procura pelo atendimento nas unidades básicas de saúde}

Em relação a procura por atendimento, é uma classe complementar a classe seis, que aborda o conhecimento dos homens sobre a UBS. A procura por atendimento está relacionada ao conhecimento desses ambientes, assim, o estudo demonstra que o conhecimento deficiente dos pesquisados sobre a atenção básica é um fator importante para a dissociação dos homens da atenção básica, conforme os relatos a seguir.

E 1: Bem, a minha ida ao posto de saúde é bem esporádica, as vezes vou por conta da vacinação do meu filho, mas além disso é muito raro por não confiar mesmo no serviço.

E 2: Raramente por que...na verdade quando a gente vai ao posto tem que ter grande necessidade de ir, ou grande problema de saúde, aí tem que ir pra deixar a saúde melhor.

E 3: Eu creio que seja um local pra cuidar da saúde das pessoas, pra descobrir alguma doença.

E 4: É um lugar onde atende as pessoas, e tem doutor, dentista, enfermeira pra atender o povo.

E 5: Pra mim é um ambiente que oferece serviços de saúde pra tratar doenças além vacinas e que tem uma proximidade com a comunidade.

A ausência do homem na atenção básica, são fatores que afunilam seu conhecimento sobre esse nível de atenção, o que determina pré-conceitos relativos a seu funcionamento e assistência, que por eles são visualizados de forma negativa. As experiências pessoas também são fatores limitantes, que ocorrem quando os homens procuram esses ambientes acompanhando algum familiar, fatores esses relacionados principalmente ao atendimento inadequado (GOMES et al., 2016).

A invisibilidade dos homens aos serviços de saúde se dá por falta de medidas de envolvimento dos mesmos. O acompanhamento das esposas grávidas durante o pré-natal contribui de forma significativa para sua sensibilização relativa a saúde. A chegada de um filho expressa sentimentos de paternidade, essa experiência pode ser vista por eles como um estimulo para o autocuidado, as estratégias desenvolvidas para abordagem paterna e sexual certamente teriam resultados significantes (MOREIRA; GOMES; RIBEIRO, 2016). 


\section{CONCLUSÃO}

Pela análise das classe formuladas, conclui-se que a acessibilidade dos homens ás Unidades Básicas de Saúde é deficiente, pois os homens possuem conhecimento superficial sobre os serviços dispensados, e a ausência ou a frequência reduzida do mesmo, assim como o acesso a medicação em farmácias o próprio preconceito, a falta do auto cuidado proveniente de atribuições socioculturais, a insatisfação e a falta de confiança na assistência da atenção primária são fatores que dificultam a procura pelos homens á Unidades Básicas de Saúde.

\section{REFERÊNCIAS}

1. ARRUDA GO, MATHIAS TAF, MARCON SS. Prevalência e fatores associados à utilização de serviços públicos de saúde por homens adultos. Ciênc. saúde coletiva. Paraná, v. 22, n. 1, p. 01-12. 2017.

2. BARBOSA CJL. Saúde do homem na atenção primária: mudanças necessárias no modelo de atenção. Revista Saúde e Desenvolvimento. Paraná, v. 6, n. 3, p 100-114. 2014.

3. BIDINOTTO DNPB, SIMONETTI JP, BOCCHI SCM. A saúde do homem: doenças crônicas não transmissíveis e vulnerabilidade social. Rev. Latino-Am. Enfermagem. São Paulo, v. 25, n. 24, p. 01-08. 2016.

4. FAROI L. Homem com "H". Ideais de masculinidade construído no marketing farmacêutico. Cad. Pagu. São Paulo, v. 40 n. 40 , p. 287-321, 2013.

5. GIL AC. Como elaborar projetos de pesquisa. 4. ed. São Paulo: Atlas, 2007. 175 p.

6. GOMES R, ALBERNAZ L, RIBEIRO CRS et al. Linhas de cuidados masculinos voltados para a saúde sexual, a reprodução e a paternidade. Ciênc. saúde coletiva. Rio de Janeiro, v. 21 n. 5, p.1545-1552. 2016.

7. MOREIRA RLSF, FONTES WD, BARBOZA TM. Dificuldades de inserção do homem na atenção básica a saúde: a fala dos enfermeiros. Esc. Anna Nery, Rio de Janeiro v.18, n. 4, p. 615-621. 2016.

8. MOURA EC, GOMES R, FALCÃO MTC et al. Mortalidade no Brasil segundo perspectiva de gênero, anos $2000 \mathrm{e}$ 2010. Rev. bras. Epidemiol. São Paulo, v.19 n. 2, p. 326-338. 2016.

9. OliveIRA, M. A. C.; PEREIRA, I. C. Atributos essenciais da Atenção Primária e a Estratégia Saúde da Família. Rev. bras. enferm. Brasília, v. 66 n. spe, p. 158-164, 2013.

10. OLIVEIRA, M. M. et al. A saúde do homem em questão: busca por atendimento na atenção básica de saúde. Ciênc. saúde coletiva. Rio de Janeiro, v. 20, n.1, p.273278, 2015.

11. RAMALHO MNA, ALBUQUERQUE AM, MAIA JKF et al. Dificuldades na implantação da política nacional de atenção integral à saúde do homem. Cienc Cuid Saude. Paraíba, v. 13, n. 4, p. 642-649, 2014.

12. SEPARAVICH MA, CANESQUI AM. Saúde do homem e masculinidades na Política Nacional de Atenção Integral à Saúde do Homem. Saúde soc. São Paulo, v. 22 n. 2, p. 415-428, 2013.

13. SEVERINO AJ. Metodologia do trabalho científico. 23. ed. São Paulo: Cortez, 2014. 223 p.

14. SILVA SO, BUDÓ MLD, SILVA MM. Concepções e práticas de cuidado na visão de homens. Texto contexto enferm. Santa Catarina, v. .22 n. 2, p. 389-396, 2013.

15. VIEIRA KLD, BORBA MR, COSTA CFS et al. Atendimento da população masculina em unidade básica saúde da família: motivos para a (não) procura. Esc. Anna Nery. Rio de Janeiro, v.17, n.1, p. 120-127, 2013. 